\title{
Comparative Mechanical Dynamic Analysis of Permanent Magnet Gen- erator Using Finite Element and Fuzzy Methods
}

\author{
Ajay Kumar, Sanjay Marwaha ${ }^{\mathrm{a}}$, Amarpal Singh* and Anupama Marwaha ${ }^{\mathrm{a}}$ \\ Department of Electronics \& Comm. Engg, Beant College of Engg. \& Technology, Gurdaspur (Punjab), India \\ ${ }^{a}$ Department of Electrical \& Inst. Engg, Sant Longowal Inst. of Engg \& Technology, Longowal (Punjab), India
}

\begin{abstract}
This paper describes the fuzzy modeling of permanent magnet generator for studying its mechanical dynamic analysis. Firstly electromagnetic torque analysis of the generator is carried out using finite element based package. Then fuzzy model of the generator is developed. Performance was evaluated by comparing, integrated fuzzy model, individual fuzzy model and finite element model for the generator. Fuzzy models were developed using adaptive neuro-fuzzy inference system (ANFIS). It is observed that system MI has a significant effect on optimal winding inductance to achieve steady state operation in shortest period of time. The winding leakage inductance should be reduced for achieving steady state operation in shortest time.
\end{abstract}

Key Words: PM generator, Fuzzy model, Finite-element analysis, Moment of inertia, ANFIS, Sugeno fuzzy inference system.

\section{INTRODUCTION}

The ability of the permanent generator to self -excite is an attractive feature that makes it a suitable choice for operation at higher power factors and efficiencies. In addition, PM machines do have the overloading capability and full torque capability at zero and at very low speeds. The understanding of the characteristics and accurate modeling of the dynamic performance of these are of fundamental importance to design engineers. Finite element analysis has been used extensively for the design and performance prediction of various types of permanent magnet machines. FE simulations for power systems are widely used to understand the behavior of the machine within the system, [1]. Modeling and control techniques based on fuzzy sets attempt to combine numerical and symbolic processing into one framework. On the one hand, fuzzy systems are knowledge-based systems consisting of linguistic if-then rules that can be constructed using the knowledge of experts in the given field of interest. On the other hand, fuzzy systems are also universal approximators that can realize nonlinear mappings. This duality allows qualitative knowledge to be combined with quantitative data in a complementary way. Compared to other nonlinear approximation techniques, fuzzy systems provide a more transparent representation of the nonlinear system under study, and can also be given a linguistic interpretation in the form of rules. The rules extracted from data can be validated by experts, and combined with their prior knowledge to obtain a complete system model describing the reality over the entire domain of interest. Very little work has been done in the area of mechanical dynamic analysis of electromagnetic devices. Until recently, the electromagnetic analysis has usually been

*Address correspondence to this author at the Department of Electronics \& Comm. Engg, Beant College of Engg. \& Technology, Gurdaspur (Punjab), India; E-mail: s_amarpal@yahoo.com confined to static representations of the machine geometry using, for example, frequency response methods in synchronous machines, as applied by [2], or slip frequency analysis in induction motors, as reported by [3]. This inevitably leads to some inaccuracy in the characterization of the machine. A complete simulation with independent dynamic electromagnetic and power system analysis has been achieved by [4], but generally this is too expensive computationally for everyday use [5], outlines improvements to the characterization of machine that can be obtained by using dynamic non-linear electromagnetic time-stepping analysis including rotation. Generation of adaptive mesh based upon nodal errors is given by [6] in which next adaptive stages are performed after updating the spacing values using previous adaptive solution. Adaptive analysis with rotation has been carried out by [7], but to take into account the rotor movement, the elements of air gap and rotor have to be modified at each rotor step. A novel algorithm of adaptive mesh generation for the nonlinear finite-element analysis of electric machines has been presented by [8]. A two-dimensional adaptive meshing technique for accurate calculation of very low cogging torque of rotating machines has been presented by [9]. But the study of effect of winding parameters on the torque is missing in all these. Simulations of PM wind turbine generators have been tested for stability by [10], but these are silent about the effect of winding parameters on the steady state performance of the generator.

In this paper firstly the finite element model of the generator is developed to carry out its electromagnetic torque analysis using a finite element based package. From the FEM simulations optimal parameters analysis of the generator for mechanical dynamic analysis is carried out. Then fuzzy model of generator is developed for its mechanical dynamic analysis and results are validated with FEM analysis to simulate its performance. 


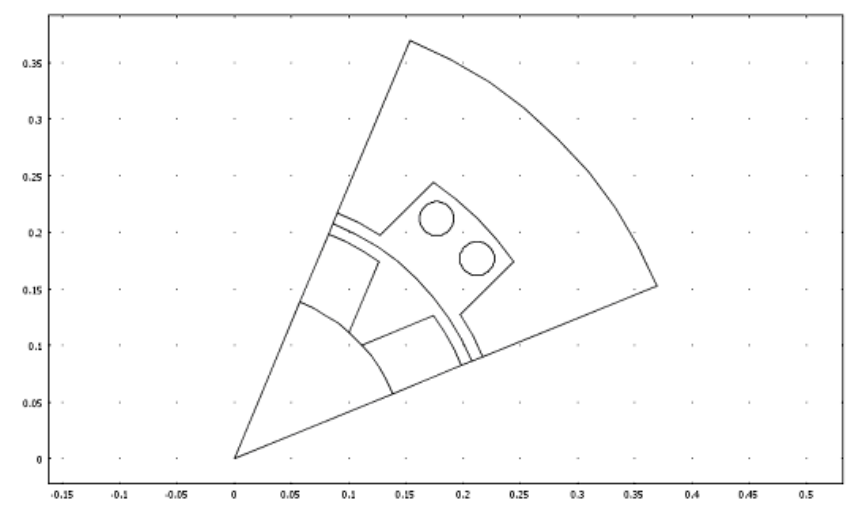

Fig. (1). Finite element model of the generator.

\section{MODELING OF PM GENERATOR}

\section{(a) Finite Element Model}

The centre of rotor consists of annealed medium carbon steel, which is a material with high relative permeability. The centre is surrounded with several blocks of permanent magnet made of samarium cobalt, creating a strong magnetic field. The stator is made up of same permeable material as the centre of the rotor. The winding is wound around the stator poles. The winding used in the stator is single turn winding. Length of the generator is $0.4 \mathrm{~m}$. Area of winding in the stator is $0.001257 \mathrm{~m}^{2}$. Relative permeability in permanent magnets is 1 . The symmetry of the generator has been exploited to reduce the model size to $1 / 8$ th of the original size. The smallest possible model of the generator is obtained by cutting radially through two adjacent poles as shown in Fig. (1).

\section{(b) Fuzzy Model}

The modeling of the system has been done using adaptive neuro fuzzy inference system (ANFIS) [11,12] by considering the input parameters; inductance and inertia of the rotor $\left(\mathrm{J}_{\text {rotor }}\right)$ and output as steady state time. This technique provides procedure to learn information about a data set, in order to compute the membership function parameters that best allow the associated fuzzy inference system to track the given input/output data. Fig. (2) shows fuzzy model of PM generator. ANFIS without subcluster is shown in Fig. (3). Fig. (4) and Fig. (5) show various membership functions of 'inductance' and $J_{\text {rotor }}$ for the model. Fig. (6) indicates the output membership function of 'steady-state time'. Here the model makes use of nine rules. Set of linguistic rules for fuzzy model without subclustering are given below:-

- If (Inductance is $\mathrm{mf} 1$ ) and ( $\mathrm{J}_{\text {rotor }}$ is $\left.\mathrm{mf} 1\right)$ then (Steady-state time is $\mathrm{mf} 1$ )

- If (Inductance is $\mathrm{mf} 1$ ) and ( $\mathrm{J}_{\text {rotor }}$ is $\mathrm{mf} 2$ ) then (Steady-state time is $\mathrm{mf} 2$ )

- If (Inductance is $\mathrm{mf} 1$ ) and ( $\mathrm{J}_{\text {rotor }}$ is $\mathrm{mf} 3$ ) then (Steady-state time is $\mathrm{mf} 3$ )

- If (Inductance is $\mathrm{mf} 2$ ) and ( $\mathrm{J}_{\text {rotor }}$ is $\mathrm{mf} 1$ ) then (Steady-state time is $\mathrm{mf} 4$ )
- If (Inductance is $\mathrm{mf} 2$ ) and ( $\mathrm{J}_{\text {rotor }}$ is $\mathrm{mf} 2$ ) then (Steady-state time is mf5)

- If (Inductance is $\mathrm{mf} 2$ ) and ( $\mathrm{J}_{\text {rotor }}$ is $\mathrm{mf} 3$ ) then (Steady-state time is mf6)

- If (Inductance is $\mathrm{mf} 3$ ) and ( $\mathrm{J}_{\text {rotor }}$ is $\left.\mathrm{mf} 1\right)$ then (Steady-state time is $\mathrm{mf} 7$ )

- If (Inductance is $\mathrm{mf} 3$ ) and ( $\mathrm{J}_{\text {rotor }}$ is $\mathrm{mf} 2$ ) then (Steady-state time is $\mathrm{mf} 8$ )

- If (Inductance is $\mathrm{mf} 3$ ) and ( $\mathrm{J}_{\text {rotor }}$ is $\mathrm{mf} 3$ ) then (Steady-state time is $\mathrm{mf} 9$ )

Fig. (7) shows the ANFIS with subclustering. Set of linguistic rules for fuzzy model with subclustering are given below:-

- If (Inductance is $\mathrm{mf} 2$ ) and ( $\mathrm{J}_{\text {rotor }}$ is $\left.\mathrm{mf} 1\right)$ then (Steady-state time is $\mathrm{mf} 1$ )

- If (Inductance is $\mathrm{mf} 2$ ) and ( $\mathrm{J}_{\text {rotor }}$ is $\mathrm{mf} 2$ ) then (Steady-state time is $\mathrm{mf} 2$ )

- If (Inductance is $\mathrm{mf} 3$ ) and ( $\mathrm{J}_{\text {rotor }}$ is $\mathrm{mf} 3$ ) then (Steady-state time is $\mathrm{mf} 3$ )

- If (Inductance is $\mathrm{mf} 4$ ) and ( $\mathrm{J}_{\text {rotor }}$ is $\mathrm{mf} 4$ ) then (Steady-state time is $\mathrm{mf} 4$ )

- If (Inductance is $\mathrm{mf5}$ ) and ( $\mathrm{J}_{\text {rotor }}$ is $\mathrm{mf5}$ ) then (Steady-state time is $\mathrm{mf5}$ )

- If (Inductance is mf6) and ( $\mathrm{J}_{\text {rotor }}$ is mf6) then (Steady-state time is $\mathrm{mf6}$ )

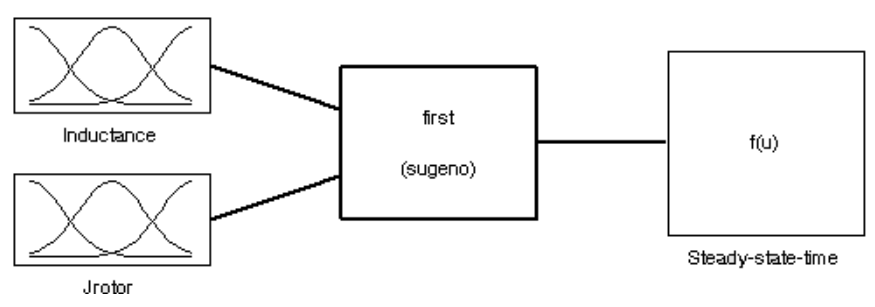

Fig. (2). Fuzzy model of the generator.

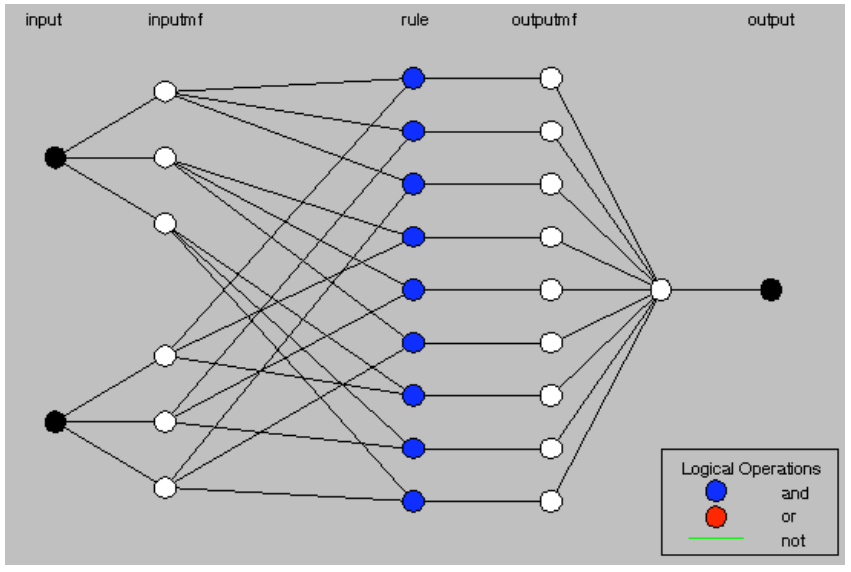

Fig. (3). ANFIS without subclster. 


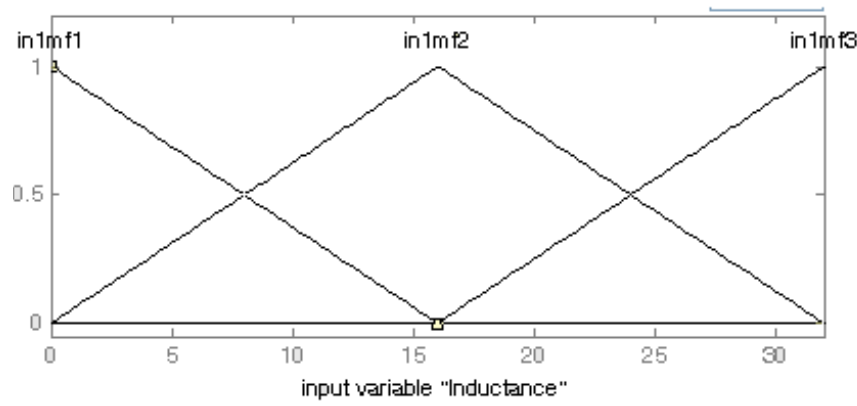

Fig. (4). Input membership function (Inductance).

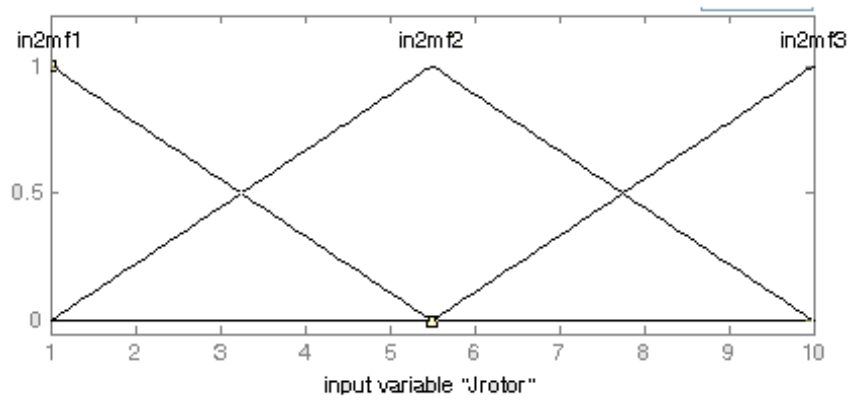

Fig. (5). Input membership function $\left(\mathrm{J}_{\text {rotor }}\right)$.

\begin{tabular}{|cc|}
\hline out1mf5 & \\
out1mf4 & out1mf9 \\
out1mf3 & out1mf8 \\
out1mf2 & out1mf7 \\
out1mf1 & out1mf5 \\
\hline
\end{tabular}

output variable "Steady-state-time"

Fig. (6). Output membership functions (Steady- state time).

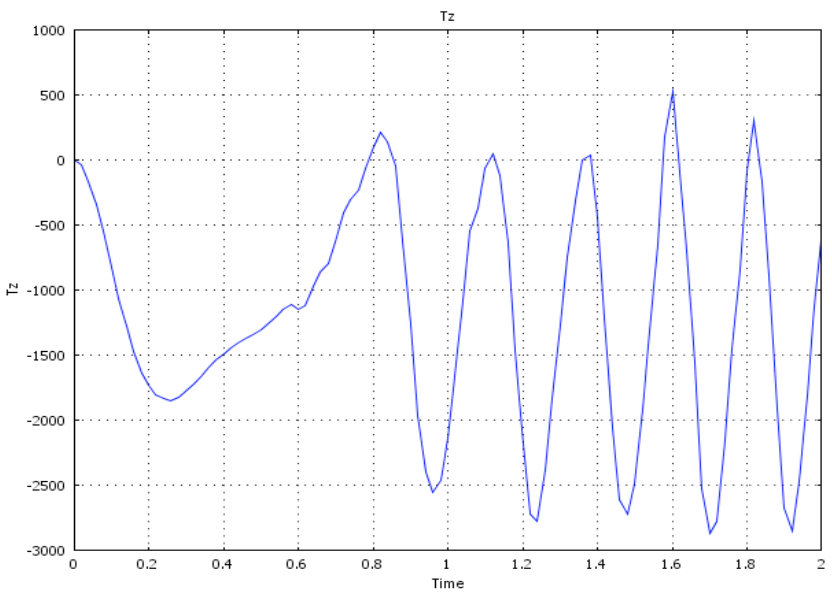

Fig. (8). Starting torque curve of the generator.

\section{RESULTS AND DISCUSSION}

The torque curve of the generator obtained using FEM during starting is shown in Fig. (8), which indicates that attenuation of transients takes a long time. This is because of larger per unit (pu) inertia of the rotor and winding inductance. For studying the effect of winding resistance and system MI with, the winding resistance, electromotive force and the MI are varied during the simulations. The steady state time is determined from the attenuation of the speed oscillation. The system MI, J, is compared with the rotor inertia $\mathrm{J}_{\text {rotor }}$ of the PM generator. The system MI has a significant effect on the optimum winding inductance to achieve steady state operation in the shortest period of time. Permanent magnets produce a pulsating magnetic braking torque during the acceleration of the rotor. This braking torque is responsible for distorting the steady state operation. This braking torque depends upon the winding leakage inductance. Hence the winding leakage inductance should be reduced for the speedy steady state operation. Table 1 shows the values of steady-state time as a function of winding inductance for different values of MI.

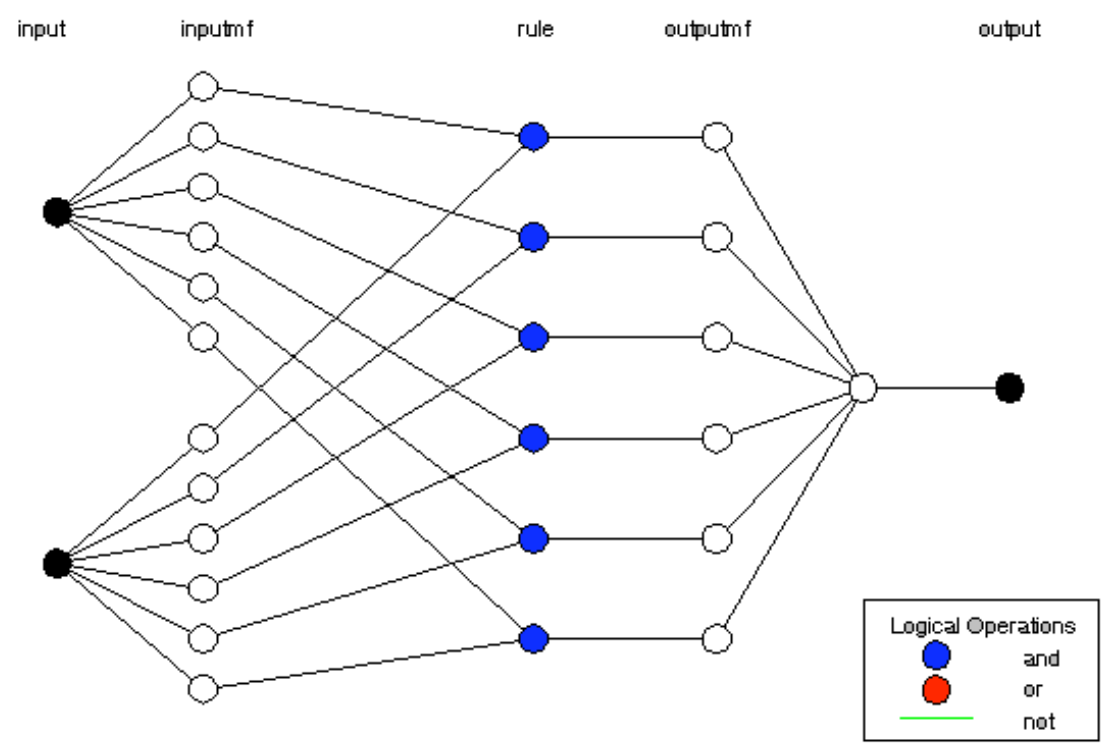

Fig. (7). ANFIS with subclustering. 
Table 1. Steady-State Time as a Function of Winding Inductance

\begin{tabular}{|c|c|c|c|}
\hline \multirow{2}{*}{$\begin{array}{c}\text { Winding In- } \\
\text { ductance } \\
(\mathbf{m H})\end{array}$} & \multicolumn{3}{|c|}{ Steady-State Time(sec) } \\
\cline { 2 - 4 } & $\mathbf{J} / \mathbf{J}_{\text {rotor }}=\mathbf{1}$ & $\mathbf{J}_{\mathbf{~}}$ rotor $=\mathbf{4}$ & $\mathbf{J} / \mathbf{J}_{\text {rotor }}=\mathbf{1 0}$ \\
\hline \hline 0 & 0.71 & 2.17 & 4.52 \\
\hline 4 & 0.76 & 2.19 & 4.54 \\
\hline 8 & 0.85 & 2.23 & 4.57 \\
\hline 12 & 0.95 & 2.27 & 4.61 \\
\hline 16 & 1.11 & 2.32 & 4.66 \\
\hline 20 & 1.31 & 2.39 & 4.72 \\
\hline 24 & 1.55 & 2.5 & 4.8 \\
\hline 28 & 1.9 & 2.65 & 4.89 \\
\hline 32 & 2.35 & 2.92 & 5.05 \\
\hline
\end{tabular}

Fig. (9) shows the rule viewers for the PM generator without subclustering for a particular case when $\mathrm{J} / \mathrm{J}_{\text {rotor }}=4$ and inductance of $16 \mathrm{mH}$ for which the output i.e. steady-state time is 2.28 seconds, which deviates from the value given in the table. Fig. (10) shows the plot of steady-state time as a function of inductance for different $J_{\text {rotor. Fig. (11) shows the }}$ rule viewers for the PM generator with subclustering for $\mathrm{J} / \mathrm{J}_{\text {rotor }}=4$ and inductance of $16 \mathrm{mH}$. The output i.e. steadystate time in this case is 2.32 seconds which is matching the value obtained by finite element analysis. Fig. (12) shows plot of steady-state time as a function of inductance for different $\mathrm{J}_{\text {rotor. With subclustering. }}$

\section{CONCLUSION}

This paper presents the mechanical dynamic analysis of PM magnet generator with combination of finite element analysis of PM generator using FEM and fuzzy logic. Firstly by using FE model, the starting torque curve of the generator is obtained. The effect of rotor MI and the optimum winding parameters on the steady state operation of the generator is the studied by FE simulations. Then fuzzy model of the gen-
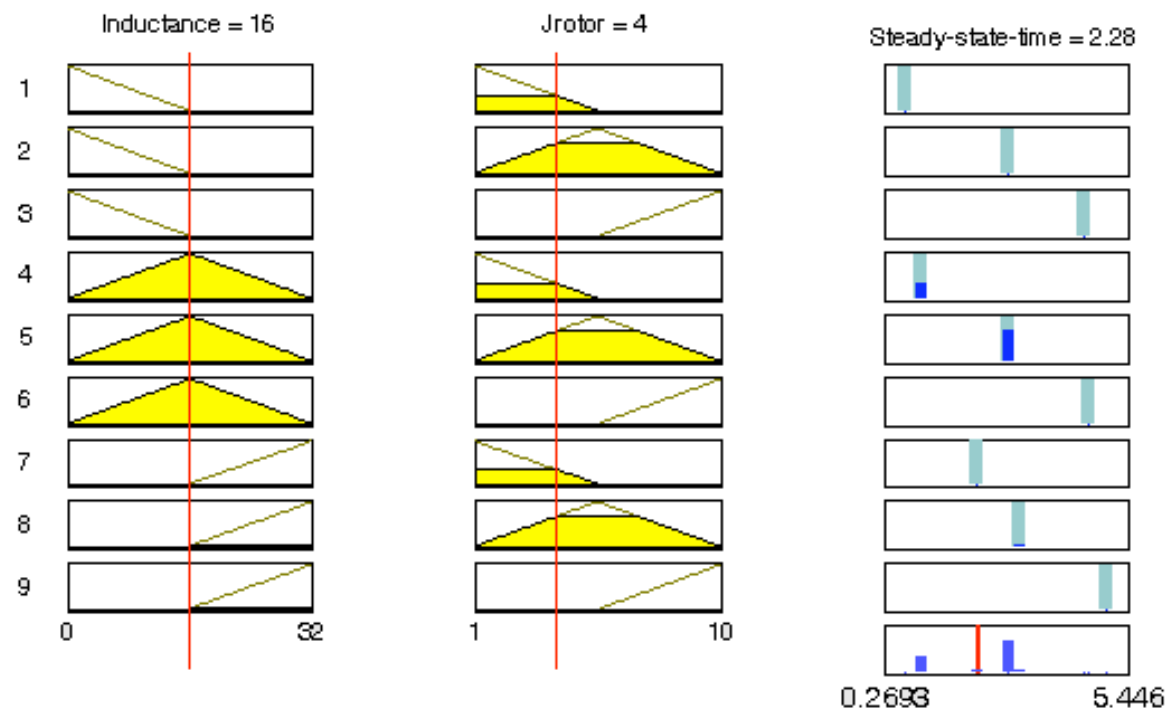

Fig. (9). Rule viewer of PM generator without subclustering.

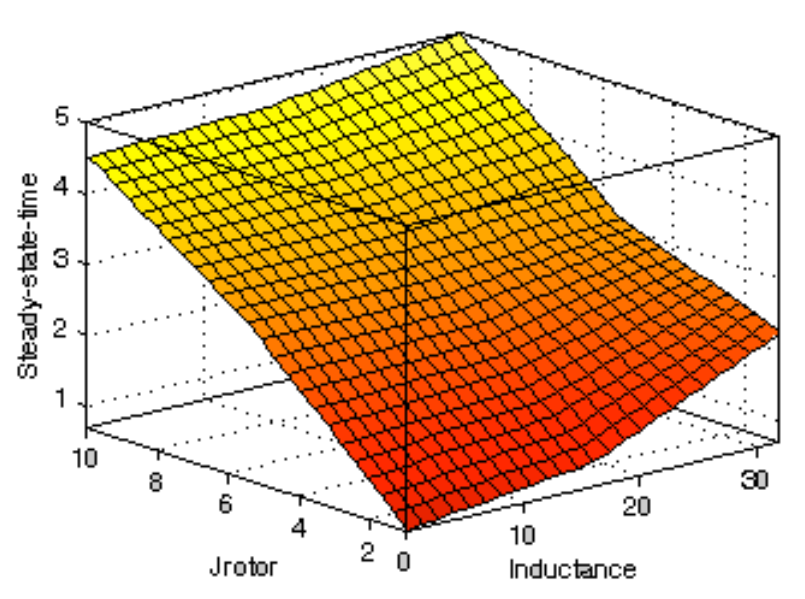

Fig. (10). Steady-State time versus winding inductance for different $\mathrm{J}_{\text {rotor }}$ without subclustering.
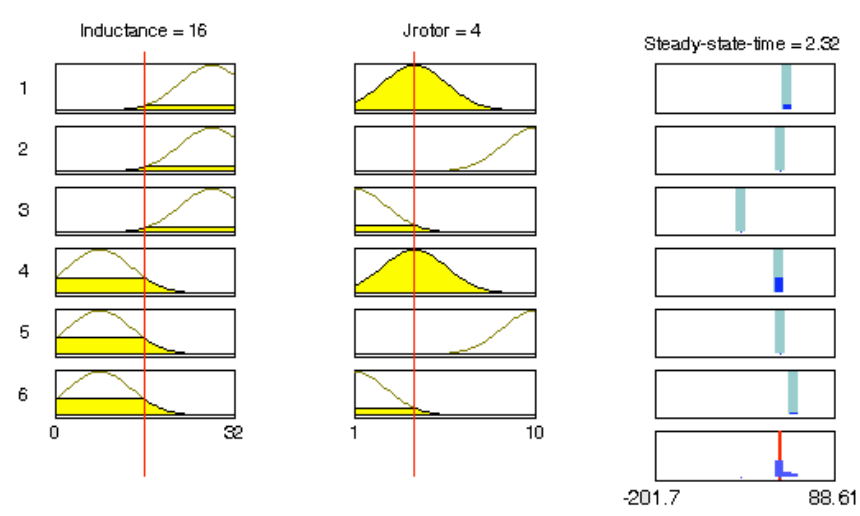

Fig. (11). Rule viewer of PM generator with subclustering.

erator is prepared for its mechanical dynamic analysis. Fuzzy model is compared with FE model. It is shown that by reducing the winding leakage inductance, the braking torque pro- 
duced by permanent magnets and hence the steady state time of the generator can be reduced. Though both finite element method and fuzzy method are very efficient and accurate, but as compared to finite element technique, fuzzy systems provide a more transparent representation of the system under study, and also give a linguistic interpretation in the form of rules. The rules extracted from data can be validated by experts, and combined with their prior knowledge to obtain a complete system model describing the reality over the entire domain of interest.

\section{REFERENCES}

[1] Dipl.-Math. Elena Zhelezina, "Adaptive Finite Element Method for the Numerical Simulation of Electric, Magnetic and Acoustic Fields", Ph.D.Thesis, University of Nuremberg-Erlangen, Germany 2005.

[2] J.W. Dougherty and S.H. Minnich, "Calculation of generator inductances by finite element methods, Sym. on Synchronous Machine Modeling for Power Sys. Studies', IEEE Power Engineering Society, 1983.

[3] S. Williamson, L.H. Lim and M.J. Robinson, "Finite element models for cage induction motor analysis", Transactions On Industry Applications, vol. 26, no. 6, pp. 1007-1017, 1990.

[4] C.R.I. Emson, C.P. Riley, D.A. Walsh, K. Ueda and T. Kumano, "Modeling eddy currents induced by rotating systems", IEEE Transactions on Magnetics, vol. 34, no. 5, pp. 2593-2596, 1998.

[5] C.S. Biddlecombe, J. Simkin, A.P. Jay, J.K. Sykulski, and S. Lepaul, "Transient electromagnetic analysis coupled to electric circuits and motion", IEEE Transactions on Magnetics, vol. 34, no. 5, pp. 3182-3185, 1998.

[6] H.S. Choi, H-S. Kim and S-Y. Hahn, "An adaptive mesh generation based on nodal errors", IEEE Transactions on Magnetics, vol. 36, pp. 1619-1622, 2000 .

[7] S. Dufour, G. Vinsard, and B. Laport," $2-\mathrm{D}$ adaptive mesh with movement" IEEE Transactions on Magnetics, vol. 37, no. 5, p. 3482, 2001.

[8] K. Yamazaki and T. Saeki, "Adaptive mesh generation within nonlinear iterative calculations for analyses of electric machines", IEEE Transactions on Magnetics, vol. 39, no. 3, pp. 1001-1004, 2003.

[9] M. Miwa, D. Dibben and T. Yamada, "High accuracy torque calculation for a rotating machine using adaptive meshing", IEEE Transactions on Magnetics, vol. 40, no. 2, pp. 1654-1657, 2004.

[10] S.K. Reddy, "Operational behavior of a double-fed permanent magnet generator for wind turbines", M.S. Thesis, Massachusetts Institute of Technology, Dept. of Electrical Engineering and Computer Science, 2005.

[11] A. Singh, A.K. Sharma, T.S. Kamal, Vishal and P. Singh, "A comparative analysis using Fuzzy modeling and OptSim on WDM systems in the presence of FWM and compensation technique", Journal of Scientific and Industrial Research (JSIR), National Institute of Science Communication and Information Resources (NISCAIR) New Delhi, India, vol. 66, pp. 339-344, 2007.

[12] J.S.R. Jang, "ANFIS: Adaptive-network-based fuzzy inference system", IEEE Transactions on Systems, Man, and Cybernetics, pp. 665-685, 1993.

(c) Kumar et al.; Licensee Bentham Open.

This is an open access article licensed under the terms of the Creative Commons Attribution Non-Commercial License (http://creativecommons.org/licenses/by-nc/3.0/) which permits unrestricted, non-commercial use, distribution and reproduction in any medium, provided the work is properly cited. 